\title{
GDFE: Geometry-Driven Finite Element for Four-Dimensional Printing
}

\author{
Tsz-Ho Kwok \\ Department of Mechanical, Industrial and Aerospace Engineering \\ Concordia University, Montreal, QC H3G 1M8, Canada \\ Email: tszho.kwok@concordia.ca \\ Yong Chen \\ Epstein Department of Industrial and Systems Engineering \\ University of Southern California, Los Angeles, CA, 90089 \\ Email: yongchen@usc.edu
}

\begin{abstract}
Four-dimensional (4D) printing is a new category of printing that expands the fabrication process to include time as the fourth dimension, and its simulation and planning need to take time into consideration as well. The common tool for estimating the behavior of a deformable object is the finite element method (FEM). However, there are various sources of deformation in $4 D$ printing, e.g., hardware and material settings. To model the behavior by FEM, a complete understanding of the process is needed and a mathematical model should be established for the structure-property-process relationship. However, the relationship is usually complicated that requires different kinds of testing to formulate such models due to the process complexity. With the insight that the characteristic of shape change is the primary focus in $4 D$ printing, this paper introduces Geometry-Driven Finite Element (GDFE) to simplify the modeling process by inducing deformation behavior from a few physical experiments. The principle of GDFE is based on the relationship between material structure and shape transformation. Accordingly, a deformation simulation can be developed for $4 D$ printing by applying the principles to the GDFEs. The GDFE framework provides an intuitive and effective way to enable simulation and planning for $4 D$ printing even when a complete mathematical model of new material is not available yet. The use of the GDFE framework for some applications is also presented in this paper.
\end{abstract}

\section{Introduction}

Additive manufacturing (AM), a.k.a. three-dimensional (3D) printing, has been developed with a growing impact to society since the invention by Charles Hull in 1984 [1]. AM has been widely used in automotive, aerospace, medical, electronics, commercial, and fashion industries $[2,3]$. Some attractive capabilities of AM include it can produce complex, freeform, and custom components without increas- ing time, material, or cost, which presents to designers "complexity for free" [4]. However, the layer-based 3D printing technology has some limitations such as slow speed, staircase effect, and difficult to fabricate lightweight thin-shell structures. Inspired by Origami [5,6], a new AM technology called four-dimensional (4D) Printing [7-10] is developed, which is roughly defined as 3D printing plus time. In other words, 4D printing is to 3D-print a structure that evolves its shape as a function of time. This demonstrates a new possibility for production and manufacturing. 4D printing is also a first glimpse into the world of evolvable materials that can respond to user needs or environmental changes. As environmental, economic, human, and other constraints continue to fluctuate, the adaptive and dynamic responses of 4D printed structures through external stimuli (e.g., heat, humidity) may make the products more resilient. 4D printing thus offers exciting opportunities for future products' design and manufacturing. It has board applications in assembly, packaging, robot actuator, drug delivery, and biological devices [11-15].

As 4D printing expands the fabrication process over time, its simulation and planning need to take time into consideration as well. However, it is challenging to control the behavior of a 4D-printed part so that it can have the desired shape-changing performance. The design can be made possible by integrating simulation into the planning phase, which allows an iterative process to update the design and estimate the physical effects of the changes. The common tool in computer-aided engineering (CAE) for estimating the behavior of a deformable object is the finite element method (FEM). However, the shape deformation comes from many different and complex factors including manufacturing process, hardware, thermal effect, material properties, etc.; some of them are even unknown. To include the factors oneby-one, we need not only a complete understanding of the manufacturing process to formulate a mathematical model, but also a set of testings to characterize the materials and 
their conditions at different stimuli as well as the interactions between them. However, such interactions are complicated that require extensive work in order to formulate such models due to the nature of the process complexity and the current limited understanding of AM technologies. For example, the 3D-printed part consists of multiple materials, and the adhesion between materials is different case-by-case. In the traditional way, the cohesive zone model parameters (and all other hardware and material characteristics) need to be first calibrated with high-precision measurement equipment before FEM can be applied.

Based on the insight that geometric shape change is the main focus of 4D printing, this paper proposes a GeometryDriven Finite Element (GDFE) method to simplify the simulation model to a geometry optimization problem. The objective of developing the GDFE framework is to enable simulation and planning for 4D printing when a complete mathematical model is not available yet for a full version of FEA. By inducing deformation behavior from a few physical experiments, the GDFE framework can convert the complicated model to a geometric optimization problem, and provide an intuitive and effective tool for utilizing new shapeshifting materials for 4D printing. The principle of GDFE is based on the relationship between material structures and shape transformation. Similar to FEM, the design domain is subdivided into a set of small elements and the deformation principles are applied on each element. The analysis on the elements are then assembled into a larger system to compute the global shape. GDFE is a sophisticated simulation platform to predict the relationship between the 4Dprinting process, the printed material structure, and the related shape transformation. This work follows a data-driven simulation framework [16] presented recently for crease-fold design. However, when material thickness and fold width are not negligible (Fig.1), the folds cannot be accurately represented as creases [17]. The modeling should include thickness in the fold, which is called thick fold here. Therefore, this research makes use of the experimental studies from a recent 4D printing system [9] to develop a thick-fold simulator using GDFE. The system [9] can quickly and easily fabricate "smart" structures with negative thermal expansion (i.e., contract upon heating). The goal of this paper is to develop an easy-to-use method to predict the behavior of shape deformation for the $4 \mathrm{D}$ printing process using thick fold. Although there are many active materials that can be used in 4D printing, studying the property of all the materials is beyond the scope of this paper. Instead, we focus on the contractionbased 4D printing system [9] because their experimental data and test cases are publicly available. Deformation principles are drawn by studying their test cases. Similar derivation could be applied to other systems and materials in future research.

$4 \mathrm{D}$ printing is a general term that describes all the structures are initially 3D-printed and then evolve over time. The shape-shifting behaviors considered in the literature [18] include 1D-to-1/2/3D, 2D-to-2/3D, and 3D-to-3D deformations. Each of which has its own importance and applications, for example, 1D cases have artificial protein structure

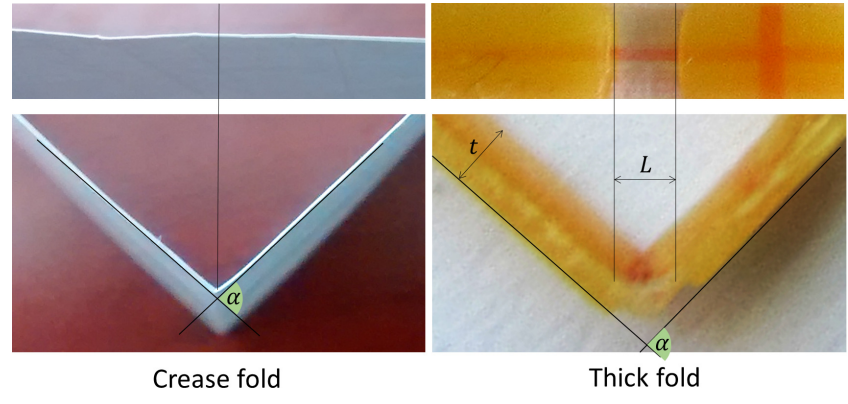

Fig. 1. Difference between a crease fold and a thick fold. Thickness $(t)$ and fold width $(L)$ are negligible (e.g., paper material) in crease fold, but not in thick fold.

and responsive smart valve to control flow, $2 \mathrm{D}$ cases have robot origami and flat-packaging, and 3D cases have smart gripper and angioplasty. In this paper, our primary application is to fabricate lightweight thin-shell structures with freeform shapes, we mainly focus our discussion in the $1 / 2 \mathrm{D}$ to-2/3D shape-shifting behaviors.

The rest of the paper is organized as follows. The related works are reviewed in this section. It is followed by the 4D printing system with material study in section 2 . The details of GDFE and the related simulation framework will be presented in section 3. Section 4 will discuss the possible use of the GDFE framework in some applications. Finally, the paper is concluded by discussion and future work in section 5 .

\subsection{Related Works}

There is research that focuses on the development of self-folding, self-assembly, or programmable materials for fabricating smarter materials and better products. A number of prototypes have been developed with different materials, fabrication technologies, and external energy sources [19]. However, many of these prototypes require an additional production step to embed the 'programmability' and the potential energy for transformation, e.g., adding magnets and elastic strands. This leads to the development of $4 D$ Printing, which streamlines the process of production for programmable and adaptive materials (see reviews [18,20,21]). The 4D printed materials are designed to deform their shapes in a pre-defined way, where the shape variation can be induced by different physical stimulation to fold, expand, shrink and curl. They can be triggered by different conditions, including temperature [7, 22], microwaves [23], or moisture content $[24,25]$, which can be used to fabricate personal and responsive products adapting to users demands, biometric information, body temperature, sweat and internal pressures. Different self-folding mechanisms have been developed in the form of planar sheets by using varying folding principles - e.g., shape memory materials [26], bilayer structures [27-29], inhomogeneous materials [30], and ShrinkyDinks films [31]. Nevertheless, the previous works mainly focus on the transformation of simple shapes, in which manual modeling was used in their construction (e.g., [8]). Recently, Raviv et al. [32] showed how to construct and simulate a complex solid structure that bends and stretches over 
time, but the work only considers a 2D grid skeleton.

A major challenge in any 4D-printed system is how to design structures that can transform from one shape into another. This requires sophisticated simulations to predict the properties between process, structure, and shape. Schenk and Guest [33] proposed a model for origami structures with elastic creased folds via truss representations. Their model is based on the introduction of torsional spring behavior at the creases. Tachi [34] used a similar approach to model the elastic behavior of sheets with creased folds by also idealizing the folds as torsional springs and solving equations of mechanical equilibrium under constraints assuring that no fold line or boundary edge is elongated. Zhu et al. [35] developed a method for analyzing surfaces under creased and bent folds. Their tool allowed for the superposition of folds with arbitrary sharpness and angle that collectively dictated the ultimate shape of the analyzed surface. Belcastro and Hull [36] used affine transformations to compute the valid origami structures and the mappings between unfolded and folded configurations. The model provided the theoretical basis for origami simulation tools, e.g., the Freeform Rigid Origami Simulator [37], to compute the unfolded patterns for a given folded target shape. Kwok et al. [16] has employed kirigami to release the surface energy by topological transform (i.e., cutting) when the given shape is highly nondevelopable. These simulation systems are mainly based on the folding that happens along the creased lines similar to the origami. However, a developable surface can be a collection of planar, cylindrical, conical, and other ruled surface sub-domains [38]. To fully utilize the capability of the programmable material, this paper presents a novel simulation framework to model more general shapes.

\section{4D Printing and Material}

At the core of the 4D printing technology are three key capabilities: the machine, the material, and the geometric 'programme'. The 4D printing machine developed by Deng and Chen [9] offers a duplex printing to fabricate smart structure, which is a sandwich structure consists of two different materials: active and passive. The active material is a prestrained polystyrene film [31], the polymer of which is frozen in its elongated state. When heat is applied, the molecular motion is increased, and the elongated polymer shrinks uniformly back to its preferred configuration. Therefore, the deformation is actually caused by the internal contraction of the active material. The printing is based on the Digital Light Processing (DLP) using light source to solidify the Acrylate resin as the passive material $[39,40]$. By the cross-linking of polymer chains, the process has a high-resolution control cost-effectively. An example of the fabrication is shown as the thick-fold case in Fig.1. Together with the contracting active material, the heterogeneous material distribution gives both structure and potential energy for folding. When a part is printed, it has an initial position, and then the active material contracts under heat, forcing the whole structure to transform. The structure reaches the final-state configuration when the active material finishes contracting. The self-
Table 1. Folding test data with different $(L, d)$ values [9]

\begin{tabular}{|l|lllllllll|}
\hline$L(\mathrm{~mm})$ & 1.50 & 3.27 & 3.42 & 3.45 & 5.49 & 5.03 & 1.80 & 3.40 & 5.08 \\
$d(\mathrm{~mm})$ & 0.11 & 0.15 & 0.12 & 0.18 & 0.14 & 0.13 & 0.24 & 0.24 & 0.24 \\
$\alpha(\mathrm{rad})$ & 1.75 & 2.27 & 2.36 & 1.70 & 2.71 & 2.88 & 0.92 & 1.44 & 1.84 \\
\hline$L(\mathrm{~mm})$ & 5.42 & 5.60 & 5.30 & 3.14 & 1.64 & 1.32 & 3.38 & 1.45 & 1.22 \\
$d(\mathrm{~mm})$ & 0.24 & 0.26 & 0.29 & 0.31 & 0.34 & 0.35 & 0.36 & 0.40 & 0.51 \\
$\alpha(\mathrm{rad})$ & 1.92 & 1.83 & 1.70 & 1.05 & 0.74 & 0.65 & 0.87 & 0.65 & 0.52 \\
\hline
\end{tabular}

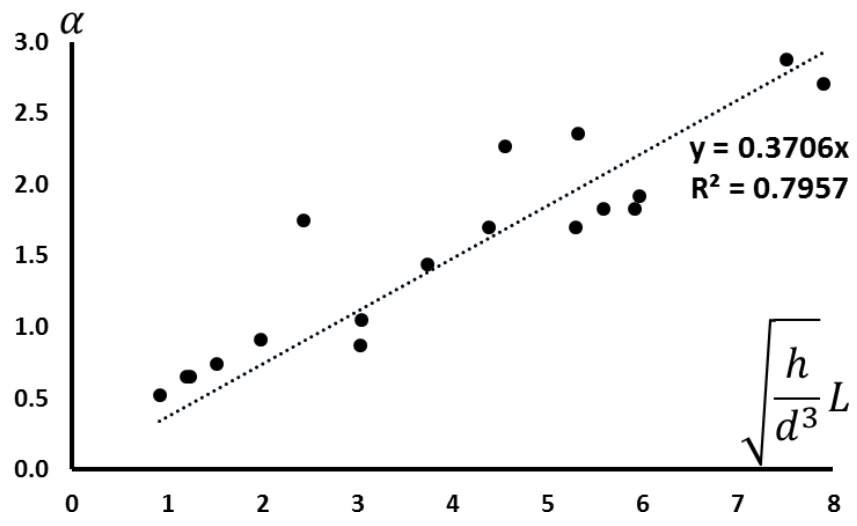

Fig. 2. The datasets in Table 1 are plotted by the fold angle against the design parameters, and a linear fitting finds $K=0.3706$.

folding mechanism is mainly based on the contraction of the film under heating with a negative thermal expansion (i.e., the expansion ratio $R<1.0$ ). The passive material does not contract under heat, and coating the passive material on the active one can alter the linear contraction of the active material. Therefore, a proper designed placement of the passive materials can create an overall anisotropy material property, which can generate shape-shifting behaviors such as bending. The spatial arrangement of the active and passive materials encompasses an embedded geometric programme to transform the structure from one shape to another with the activation energy.

\subsection{Experimental study}

Deng and Chen [9] have studied the material performance under thermal stimuli on the crease-fold designs, and found that there is difference between small and large fold angles. Thus, they modeled the deformation for these two cases separately. Their assumption is that a small fold angle is caused by elastic deformation while a large fold angle is caused by plastic deformation. However, this assumption is only valid for creased fold. The reason for different behaviors of small and large fold angles is that the thickness of the material is not considered in the crease-fold design. A thick fold forms a fold angle $(\alpha)$ is based on its fold width $(L)$. The larger the fold width is given, the larger the fold angle can be achieved. In other words, a large fold angle is formed by a long fold with consecutive small deformations, instead of a large plastic deformation. Therefore, this pa- 
per reanalyzes the experimental data reported in their paper and derives deformation model for thick-fold designs. To be complete, their folding test data are included in Table 1, where $d$ is the thickness of the passive material.

Following their idea in modeling the deformation of the passive material as large deflections of a wide beam [9], the deformation is caused by the energy generated in the contraction of the active material, and thus the relationship between the fold angle $(\alpha)$ and the design parameters (e.g., fold width: $L$, the thickness of active and passive materials: $h, d$ ) can be summarized by

$$
\alpha=K \sqrt{\frac{h}{d^{3}}} L
$$

where $K$ is a coefficient related to the temperature and material properties. Instead of finding all the parameters to calculate $K$, we can treat it as an constant in a similar manufacturing pipeline and calibrate its value by the physical experiments done by Deng and Chen [9]. As the thickness of the active material is constant $(h=0.29 \mathrm{~mm})$, the experiment was done by testing different values for fold width $(L)$ and thickness of passive material $(d)$. The data in Table 1 are plotted in Fig. 2 by the fold angle $(\alpha)$ against the design parameters $\left(\sqrt{\frac{h}{d^{3}}} L\right)$, and a best-fit straight line is drawn on the data sets. The fitting is reasonably good having the coefficient of determination around 0.8 , and it is found that $K=0.3706$. Here, only 18 experiments are conducted to build the mathematical model for the deformation. If the thickness of passive material $(d)$ is also fixed, the number of testings can be further reduced.

\subsection{Deformation principle}

After finding the relationship between the fold angle and the design parameters, the next question is how it can be used in modeling thick folds. The hinge-based design of Deng and Chen [9] is actually a special case in thick-fold design that maximizes the fold angle with a given fold width (i.e., maximized curvature), where one side of the hinge is coated with passive material while another side is empty with no passive material. To generalize the design for arbitrary curvature or freeform shape, the underlying deformation principle of material should be induced. Here, as the deformation is caused by the contraction of material under heat, the major principle is the ratio of negative thermal expansion $(R)$. Therefore, our goal here is to find out the value of $R$ from the hinge-based design.

Refer to the notation in Fig. 3, a hinge of length $L$ is composed of an active layer and a passive layer with thickness $h$ and $d$, respectively. The passive layer constrains one side of the active layer from contraction, and thus only another side of the active layer contracts. The contraction of material creates a moment to bend the hinge. The resultant fold angle is directly related to the length of hinge, the thickness of layers, and the contraction ratio. To calculate the expansion ratio $R$, the bending result is approximately described as a circular arc, so that the concepts from geometry

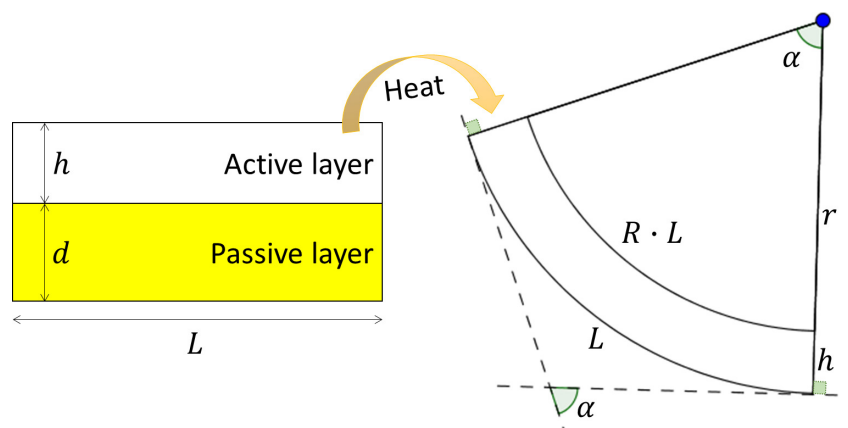

Fig. 3. When an active material contracts in one side by a shrinkage ratio $R$ with another side constrained by a passive material, it selftransforms to an arc under heat with a bending angle $\alpha$.

can be applied. Assume the fold angle is $\alpha$, the hinge forms an arc of length $L$ with radius $r+h$ and subtending an angle $\alpha$ with a center (Fig. 3 right). The inner arc is of a smaller radius $r$ and having a length of $R \cdot L$, where $R$ is the expansion ratio. From geometry, the arc length equals to the central angle times the radius, i.e.,

$$
L=\alpha(r+h) \quad \text { and } \quad R \cdot L=\alpha r
$$

By eliminating $r$, the ratio $R$ can be expressed as

$$
R=1-\frac{\alpha h}{L}
$$

Substituting Eq.(1) to Eq.(2), it becomes

$$
R=1-0.3706\left(\frac{h}{d}\right)^{1.5}
$$

It can be seen that $R$ is actually a variable of the thickness of the active and passive layers $(h, d)$, and it will be used for the development of simulation.

\section{Geometry-Driven Finite Element}

An important component for the viability of 4D printing is the design and placement of the passive material that embeds the complex state-changing capability directly into the heterogeneous material in the designed structure. Instead of constructing hinges to create the crease-fold design resulting in piecewise linear surfaces, this paper proposes GeometryDriven Finite Element (GDFE) to generalize the crease-fold design to smooth curvatures and general shapes. Making use of the deformation principle induced from the previous section, it can be converted to a geometric optimization problem assuming model is fabricated by the aforementioned manufacturing process. A sophisticated simulation can then be developed to predict the deformation behavior of the printed part with heterogeneous material distribution.

Similar to the concept of FEM, a hinge of length $L$ can be subdivided into a set of small elements, i.e., $L=n l$ where 


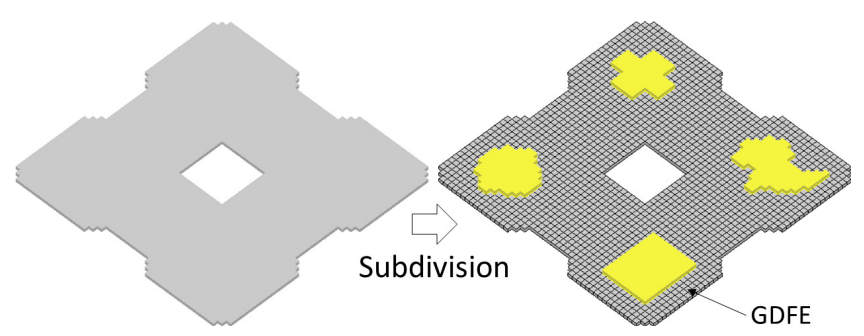

Fig. 4. Similar to FEM, the GDFE framework subdivides the design domain into a set of GDFEs, so that the deformation principle is applied locally in each element.

$n$ is the number of cells with length $l$. The expansion ratio $R$ can also be seen as applying on each small element separately, i.e., $R \cdot L=R \cdot n l=n(R \cdot l)$. When the elements are sufficiently small, the contraction and deformation can be approximated linearly. Therefore, a whole design domain can be subdivided into a set of elements (Fig. 4), and a simpler case is considered locally in each of the elements. Given the expansion ratio $R$, the final shape of each element can be computed based on its own configuration (refer to the bottom of Fig. 5 for an illustration in 2D). One can see that the final shapes of the element are computed geometrically based on $R$, which is calculated from the experimental data. Therefore, the small cell is named Geometry-Driven Finite Element (GDFE) in our research. Based on the possible configurations, they can be summarized into two types of GDFE:

Rigid GDFE (R-GDFE): both sides of this element are coated with the passive material. This element will stay in same shape throughout the transformation.

Contract GDFE (C-GDFE): one side of this element is empty and another side is coated with the passive material. The empty side will contract linearly by $R$.

The GDFE with both sides empty can be treated as a special case of R-GDFE that starts with longer material and uniformly shrink throughout the transformation. Although it may provide extra design flexibility, this type of GDFE is not discussed explicitly in this paper.

\subsection{Simulation}

With the definition of GDFE, this section presents a simulation framework using GDFE. The formulation used in the simulation is derived based on each GDFE in the design domain, and a GDFE here is represented by (but not limited to) a hexahedron defined by eight vertices $\mathbf{V}_{\mathbf{i}}=\left(v_{i, 1}, \ldots, v_{i, 8}\right)$, where the subscript $i$ indicates that they are from the $i$-th element. As the active material contracts uniformly in a plane when heat is applied, the expansion ratio $R$ in Eq.(3) can be generalized for $2 \mathrm{D}$ cases. Therefore, the final shape of a GDFE can be computed based on its rigid or contract configuration, and it is also represented by eight vertices $\mathbf{V}_{\mathbf{i}}^{\mathbf{f}}=\left(v_{i, 1}^{f}, \ldots, v_{i, 8}^{f}\right)$. The algorithm of simulation is described as follows. First, the optimal orientation of each GDFE is determined according to its current shape $\mathbf{V}_{\mathbf{i}}$ and the final shape $\mathbf{V}_{\mathbf{i}}^{\mathbf{f}}$, i.e., finding a rotational matrix to align $\mathbf{V}_{\mathbf{i}}^{\mathbf{f}}$ to $\mathbf{V}_{\mathbf{i}}$. This is the geometric optimization that can be formulated as

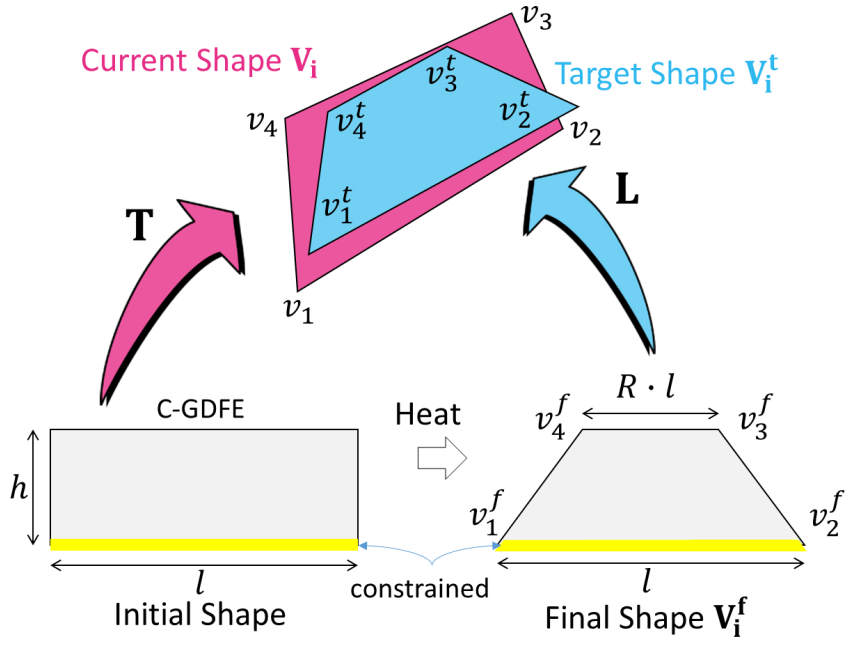

Fig. 5. The final shape of a C-GDFE can be computed by linear contraction on one side while another side is constrained. It is used to compute the target shape and optimize the current shape to it.

a minimization of the difference between the current and final shapes. After that, they are then assembled into a larger system that models the entire domain for the whole structure. Final, the orientation step and the assembly step are iterated, and the solution can be efficiently approximated by minimizing the aforementioned error for each GDFE.

\section{Orientation step}

Given the current shape $\mathbf{V}_{\mathbf{i}}=\left(v_{i, 1}, \ldots, v_{i, 8}\right)$ and the final shape $\mathbf{V}_{\mathbf{i}}^{\mathbf{f}}=\left(v_{i, 1}^{f}, \ldots, v_{i, 8}^{f}\right)$ for a GDFE, this step is to find a rotational matrix for computing the target position $\mathbf{V}_{\mathbf{i}}^{\mathbf{t}}=$ $\left(v_{i, 1}^{t}, \ldots, v_{i, 8}^{t}\right)$ to construct the orientation equation (see Fig. 5 for an illustration in 2D). First, a linear transformation between the final and the current shapes can be defined by a transformation matrix $\mathbf{T}$ and a displacement vector $\mathbf{d}$ as $\mathbf{T} \mathbf{V}_{\mathbf{i}}^{\mathbf{f}}+\mathbf{d}=\mathbf{V}_{\mathbf{i}}$. There are eight vertices and this system is overdetermined, so $\mathbf{T}$ is computed by least square method and results in an affine transformation including both the scaling and the rotation. To find the optimal orientation for the target shape, the pure rotation has to be extracted from T. One can decouple the scaling and the rotation by the singular value decomposition (SVD) into $\mathbf{T}=\mathbf{U} \Sigma \mathbf{V}^{\top}$. As $\mathbf{T}$ is a square matrix with positive determinant, $\mathbf{U}$ and $\mathbf{V}$ are the rotational matrices, and $\Sigma$ is a scaling matrix. Then, the pure rotational matrix is $\mathbf{L}=\mathbf{U V}^{\top}$. Final, the target shape of the GDFE is computed by $\mathbf{V}_{\mathbf{i}}^{\mathbf{t}}=\mathbf{L} \mathbf{V}_{\mathbf{i}}^{\mathbf{f}}+\mathbf{d}$.

\section{Assembly step}

The orientation step computes the target shapes $\mathbf{V}_{\mathbf{i}}^{\mathbf{t}}$ for all the GDFE, and they are used to formulate the equations that will be assembled into a larger system (the whole structure). To minimize the difference between the current shape $\mathbf{V}_{\mathbf{i}}$ and the target shape $\mathbf{V}_{\mathbf{i}}^{\mathbf{t}}$ of a GDFE, an energy function is developed for the simulation:

$$
E_{i}=\Delta_{i}\left\|\mathbf{D V}_{\mathbf{i}}-\mathbf{D} \mathbf{V}_{\mathbf{i}}^{\mathbf{t}}\right\|_{2}^{2}
$$


where $\Delta_{i}$ is the volume of the GDFE, and $\mathbf{N}$ is a $8 \times 8$ matrix in which the off-diagonal elements are all $-\frac{1}{8}$ while the main diagonal entries are $\frac{7}{8}$, i.e., $\mathbf{D}=\left(d_{i, j}\right)$ is defined as

$$
d_{i, j}=\left\{\begin{array}{rl}
7 / 8 & \text { if } i=j \\
-1 / 8 & \text { if } i \neq j
\end{array} \quad \forall i, j \in\{1,2, \ldots, 8\}\right.
$$

For an example, expanding $\mathbf{D} \mathbf{V}_{\mathbf{i}}$ for the first vertex is

$$
\frac{7}{8} v_{i, 1}-\frac{1}{8} \sum_{j=2}^{8} v_{i, j}=v_{i, 1}-\frac{1}{8} \sum_{j=1}^{8} v_{i, j}
$$

where $\frac{1}{8} \sum_{j=1}^{8} v_{i, j}$ is the average (center) of $\mathbf{V}_{\mathbf{i}}$. Therefore, the effect of $\mathbf{D}$ is to shift the center of the GDFE to the origin eliminating the translational constraint, so that the translation can be computed implicitly during optimization. This has a great improvement to the speed of convergence.

As $\mathbf{V}_{\mathbf{i}}^{\mathbf{t}}$ is defined from the orientation step, $\mathbf{D} \mathbf{V}_{\mathbf{i}}^{\mathbf{t}}$ is known and the only unknown is $\mathbf{V}_{\mathbf{i}}$. Comparing FEM with Eq.(4), it is possible to view $\mathbf{D}$ as the element stiffness matrix, and $\mathbf{D V}_{\mathbf{i}}^{\mathbf{t}}$ as the element force vector. Let $\mathbf{V}$ be a vector that stacks all the vertices $\left(v_{1}, \ldots, v_{n}\right)$ from the whole design domain, where $\mathbf{V}_{\mathbf{i}} \subseteq \mathbf{V}$, and there are $m$ GDFEs. Assembling Eq.(4) from all $m$ GDFEs, the master system for the optimization can be written as:

$$
E=\sum_{i=1}^{m} \Delta_{i}\left\|\mathbf{D} \mathbf{V}_{\mathbf{i}}-\mathbf{D} \mathbf{V}_{\mathbf{i}}^{\mathbf{t}}\right\|_{2}^{2}=\|\mathbf{A V}-\mathbf{p}\|_{2}^{2}
$$

where $\mathbf{A}$ is a $8 m \times n$ matrix combines all the mean-centered matrices and $\mathbf{p}$ is the assembly of all the projections from the target shapes. By setting the derivative of Eq.(5) equals to zero, it can be solved as $\mathbf{A}^{\top} \mathbf{A V}=\mathbf{A}^{\top} \mathbf{p}$ to update $\mathbf{V}$. Remarked that $\mathbf{A}$ depends only on $\mathbf{N}$, which never changes, so $\mathbf{A}^{\top} \mathbf{A}$ can be pre-factorized and reused for all the iterations. Hence, the solving is efficient with only backward substitutions. $\quad \mathbf{A}^{\top} \mathbf{A}$ is a $n \times n$ matrix instead of $3 n \times 3 n$ as used in FEM, because the three degrees of freedom (DOF) of a vertex are decoupled in GDFE, and they can be computed separately with the same master matrix.

The simulation is dynamics, and the geometric optimization alternates these two steps: orientation and assembly, to approach the final solution. The alternating optimization scheme for each iteration is summarized as: (1) For given $\mathbf{V}$, find the orientation for each GDFE to compute and assemble the projection vector $\mathbf{p}$; and (2) For given $\mathbf{p}$, solve $\mathbf{A}^{\top} \mathbf{A V}=\mathbf{A}^{\top} \mathbf{p}$ for $\mathbf{V}$ by backward substitution.

\subsection{Validation}

To validate the correctness of the simulation based on GDFE, two types of validation have been performed. The first type is to compare the simulation result with the anglewidth relationship found by the experimental study in Eq.(1).
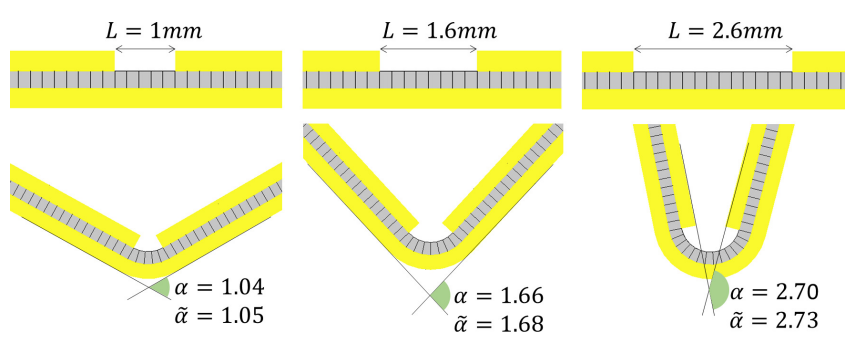

Fig. 6. The GDFE framework is validated by comparing the simulated bending angle $\alpha$ with the calculated angle $\tilde{\alpha}$ by the analytical model in Eq.(1). Different fold widths $L$ are tested, and the results show good matches.

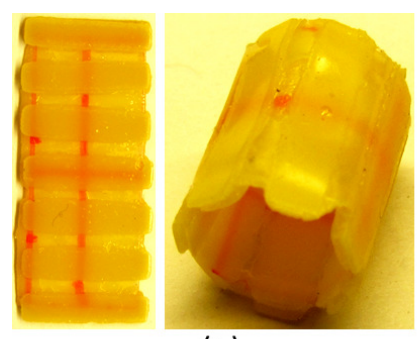

(a)
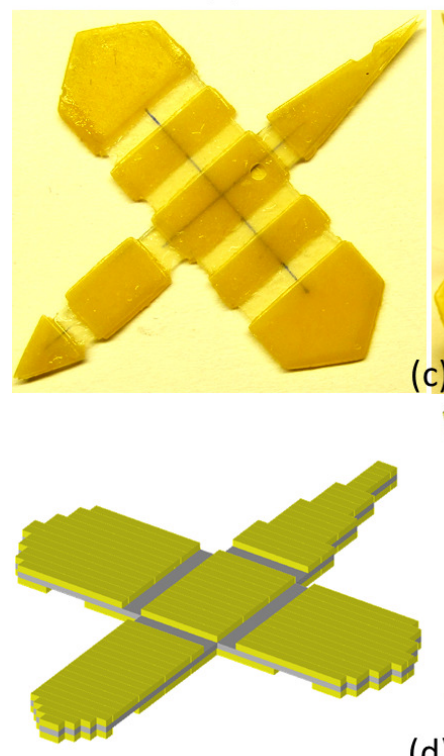

(d)

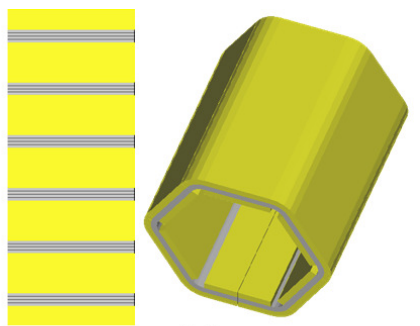

(b)
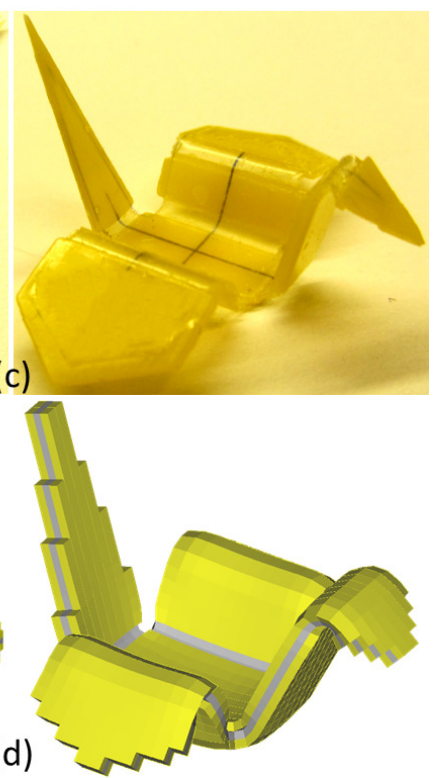

Fig. 7. The test cases (Courtesy Deng and Chen [9]) in (a) and (c) are reconstructed by the GDFE simulator in (b) and (d). The red and black lines are just used to facilitate alignment during fabrication without any effect on the deformation.

Three cases are tested: $L=\{1,1.6,2.6\} \mathrm{mm}$, and the presented GDFE framework is applied to simulate the folded shapes (Fig.6). The fold angles are measured for all three cases: $\alpha=\{1.04,1.66,2.70\} \mathrm{rad}$. The thicknesses of the active and passive layers used in practice [9] are $h=0.29 \mathrm{~mm}$ and $d=0.33 \mathrm{~mm}$, so the ratio of negative thermal expansion in Eq.(3) is $R=0.7$. Then, the calculated angle by Eq.(1) are $\tilde{\alpha}=\{1.05,1.68,2.73\} \mathrm{rad}$. It can be seen that the presented GDFE framework can simulate the folding with the maximum error of only $0.03 \mathrm{rad}$ for an angle close to $\pi$. 


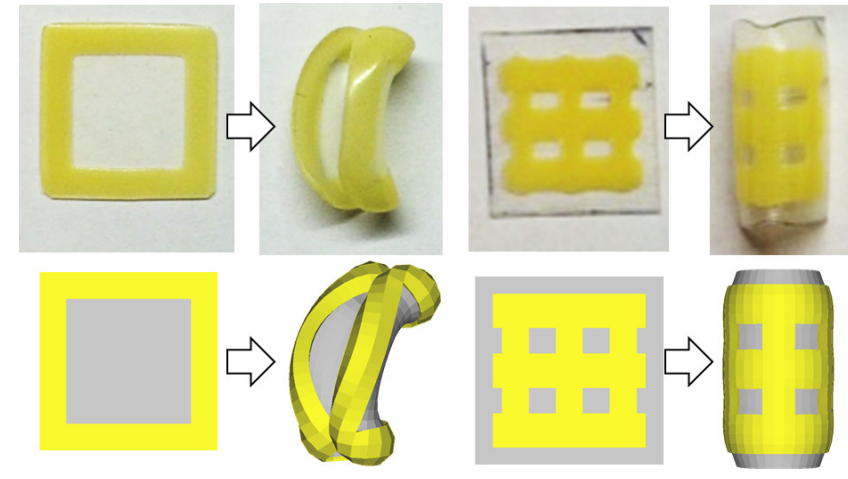

Fig. 8. Comparison between the physical fabrication and the simulation result by GDFE on two freeform surfaces.

The second type of validation is to reconstruct the test cases shown by Deng and Chen [9] using the GDFE simulator. Two cases are picked including a rolled tube and a crane design. The same settings are used to design the unfolded patterns, and the GDFE framework does the deformation simulation. The simulated shapes match well with the physical test cases as shown in Fig.7. All the test cases validate that the GDFE simulator developed based on the physics of 4D printing process can capture the contraction-based shape deformation. As a future work, we will quantitatively measure the accuracy and tolerances with precision 3D scanning instruments.

\section{Application}

Based on the GDFE simulation system, it can predict the behavior of more complex deformation and has the potential to plan the material distribution in the $4 \mathrm{D}$ printing process. This section discusses some applications as well as the related extensions that are enabled by the GDFE simulator.

\subsection{Freeform shape}

GDFE framework is an element-based simulation. It can simulate the crease-fold design (e.g., Fig.7); in addition, it can also be used to simulate freeform shape deformation. Passive materials can be specified element-by-element, and it is possible to distribute the Rigid (R-GDFE) and Contract GDFEs (C-GDFE) in a more intelligent way. For example, Fig. 8 shows two different 2D patterns that are used as the passive layer. Upon heating, they can achieve curvy and smooth freeform shapes that are very difficult to achieve by crease folding. This shows a great opportunity in designing freeform and complex shapes based on the 4D printing process, and this framework is an effective tool for the digital material design on $3 \mathrm{D}$ printed structures.

\subsection{Design for 4D printing}

The GDFE-based simulation solves a forward problem that determines the final shape with given material structures and deformation principles. Based on it, we can now work towards a solution for another major challenge in the $4 \mathrm{D}$ printing system, which is how to design the material structure that can be transformed into a desired shape. This is an inverse problem, which is the determination of the material distribution in a structure based on the final desired shape and the deformation principle. Such a reverse problem is critical for 4D printing despite it is quite challenging. This paper presents a preliminary study on this inverse problem using the GDFE-based simulator for one-dimensional (1D) case. Borrowing the concept from Fig.3, an arc can be represented by a given length $L$ and curvature $1 / r$. Given the input $(L, r)$, the angle is $\alpha=L / r$ and the contracted length is $\Delta L=$ $L-R \cdot L=\alpha h$. In order to achieve the contracted length, the required length of the C-GDFEs is $L_{e}=\alpha h /(1-R)$. Figure 3 is actually the case of maximum curvature as explained before, where an arc is constituted totally by C-GDFE. If a lower curvature $(1 / r)$ is desired with the same length, i.e., $L_{e}$ is smaller, both C-GDFEs and R-GDFEs should be incorporated, where the length of C-GDFEs must equal to $L_{e}$ and should be distributed uniformly and alternated with RGDGEs along the whole length $L$. Therefore, an input of the couple $(L, r)$ can define a curve segment with a length $L$ and curvature $1 / r$. By inputting a series of couple $\left(L_{i}, r_{i}\right)$, the final shapes and the patterns can be generated. The examples of some English letters (e.g., "Concordia") are shown in Fig.9. All the letters are generated by a maximum of three input couples.

To generalize the design to more freeform shape, e.g., the hand sketching letter ' $\mathrm{S}$ ' shown as the blue line in Fig. 10, the shape has to be segmented and approximated by a sequence of geometric primitives - e.g., straight line and circular arc. Let $p_{i}$, where $i=0, \ldots n$, be the vertices of the input shape, the segmentation is done as following:

1. For vertex $p_{i}$, find its tangent $\tau_{i}$ by $p_{i-1}$ and/or $p_{i+1}$.

2. Build a graph $\tau(s)$ with the $y$-axis is the angle $\tau_{i}$ and $x$ axis is the distance $s_{i}$ calculated from the beginning of shape $p_{0}$ to the current vertex $p_{i}$.

3. Segment graph $\tau(s)$ into $m$ straight segments by minimizing a functional $F(m, \Delta)=\sum_{j=1}^{m} \varepsilon_{j}+m \cdot \Delta$, where $\varepsilon_{j}$ is a summed square residual of the approximation for the $j$-th segment, and $\Delta$ is the penalty for each segment as a control for the number of segments. (See [41] for a detail explanation)

4. For a straight segment on $\tau(s)$, the differences in the $x$ and $y$-axes define the length and curvature of its corresponding circular arc, or a straight line if there is no difference in the $y$-axis.

5. Each segment results in a couple $\left(L_{i}, r_{i}\right)$, and the collection of them is inputted to generate the approximated shape using the method described above.

There are three levels of approximation for the handsketching ' $S$ ' shown in Fig.10. The left of the figure shows the graphs $\tau(s)$ as "Orig" with the approximated straight segments as "Approx", and the right shows the comparison of the sketching and the approximated shapes generated by the couples converted from the straight segments. In the first level of approximation, $\Delta=1$ is used, and it is approximated by two segments, i.e., $m=2$. Therefore, the shape is approx- 


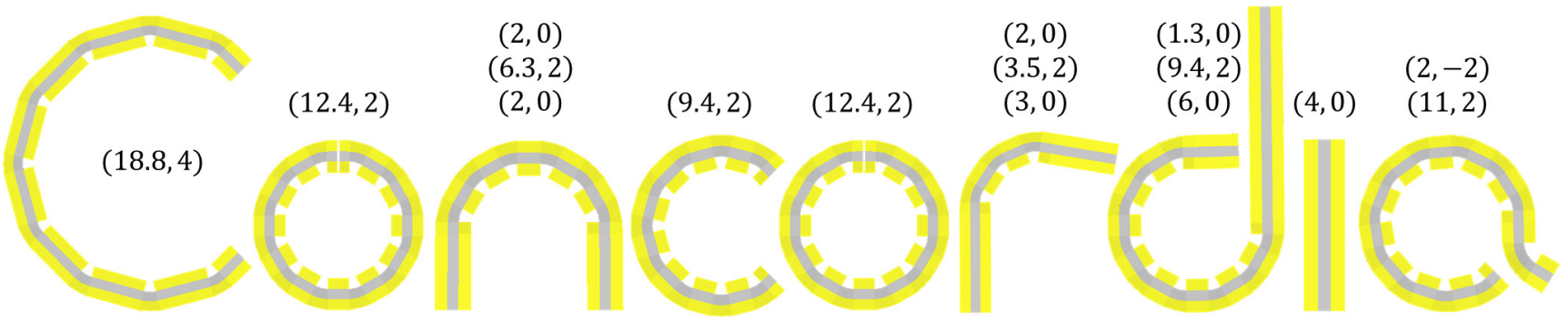

Fig. 9. By inputting a series of couples $\left(L_{i}, r_{i}\right)$, the corresponding letters can be generated. $L$ is the length, and $r$ is the radius for the circular arc ( $r=0$ means straight). Positive value of $r$ means folding in anticlockwise direction, and negative value folds in clockwise.
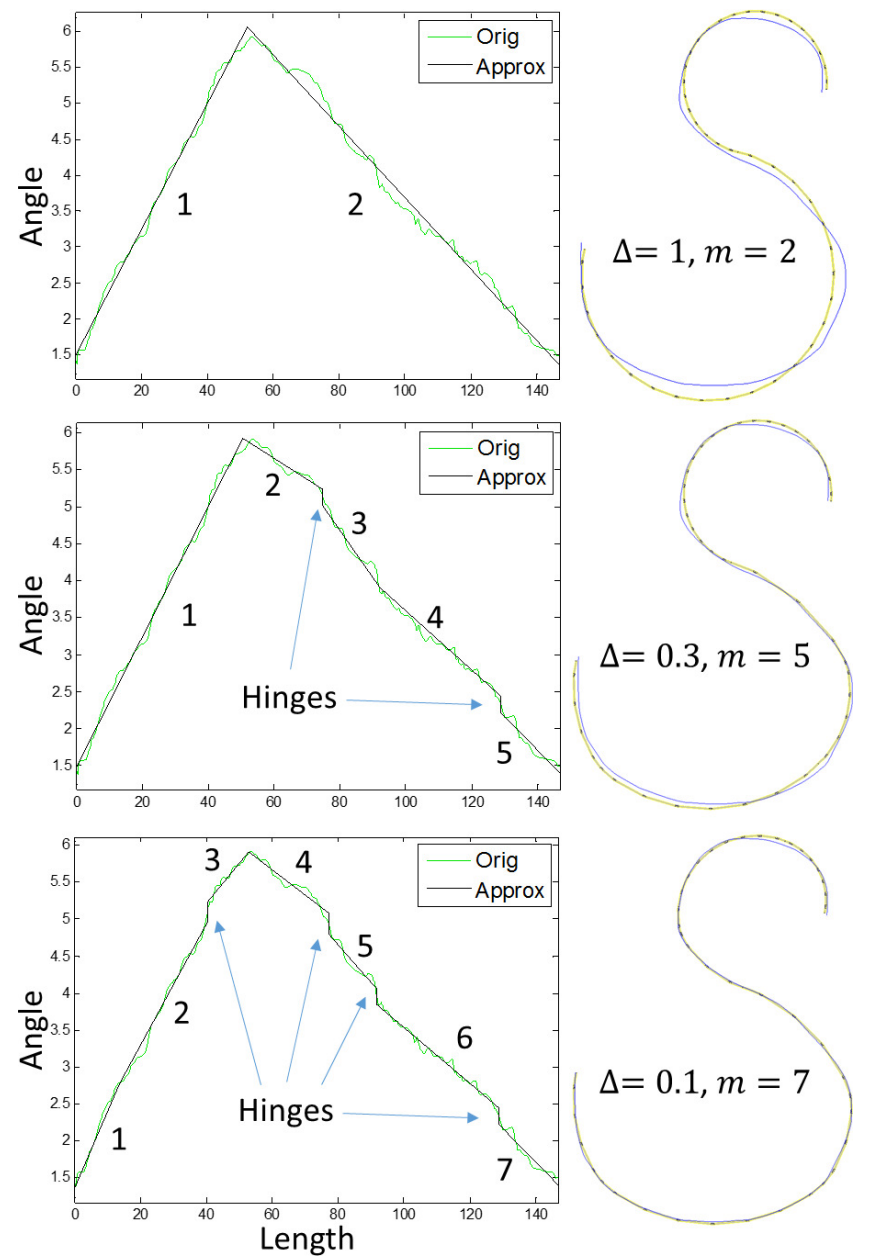

Fig. 10. A hand-sketched letter ' $S$ ' is reproduced by $4 \mathrm{D}$ printing. Increasing the levels of polyline fitting on the tangent graphs can improve the shape approximation.

imated by two circular arcs with different radius and lengths. With the higher level of approximation $\Delta=0.3$ and 0.1 , there are more segments $m=5$ and 7 for the approximation. When the orientation of the neighboring circular arcs are not compatible, the straight segments on $\tau(s)$ are connected by a vertical line, which means there is a sharp change in angle at a particular point without length change. These are the hinges that can only be achieved by creased fold, and it is approximated by the maximized curvature thick fold here. Fortunately, the magnitudes of the sharp changes are not large, and

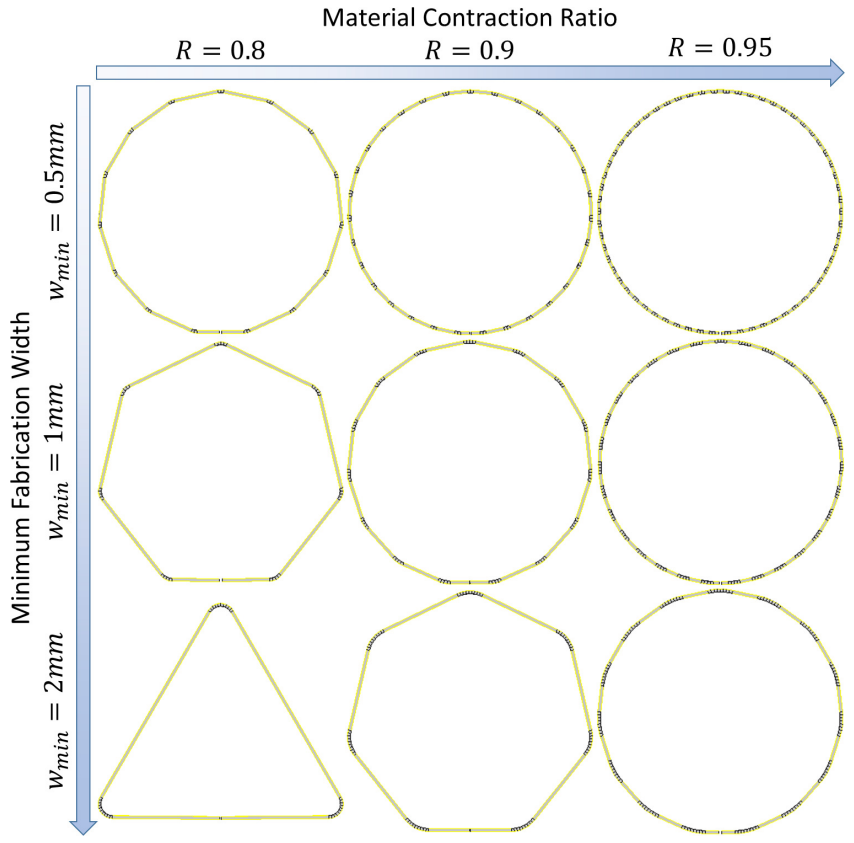

Fig. 11. All the results here are approximating a same circle, but different results are obtained with different properties of hardware (minimum fabrication width) and material (contraction ratio).

the approximation in the third level fits well to the sketched shape.

\subsection{Design Validation}

Ideally, if there is an infinite number of infinitely small GDFEs, the curves can be infinitely smooth (i.e., the higher resolution, the better approximation). However, depends the hardware, there is always fabrication limitation that leads to an approximation error. While a design can be very complicated, the fabricated result may be different. The design has to be validated before fabrication. The presented GDFEs framework and the design for $4 \mathrm{D}$ printing can be used to visualize the deformed shapes. Therefore, it can help designers to understand how good an approximation is based on a heterogeneous material design.

For example, there are two factors studied in Fig.11: the minimum size that can be fabricated $w_{\min }$ by hardware and the expansion ratio $R$ of the given material. All the tests are to fabricate a same circle, but they have different approximated 
results. When $w_{\text {min }}$ is small, it can achieve a higher resolution, and thus the circle can be smoother in shape. Therefore, it is always better if the printer has a higher resolution. On the other hand, when $R$ is large, it means the material has low contraction rate, and thus it needs a larger length $L_{e}$ of C-GDFEs to achieve the same contraction length. Although larger $L_{e}$ can give a smoother shape, there is a trade-off that the minimum feature size that can be fabricated by the $4 D$ printing process is also larger. However, if $R$ is small and $L_{e}$ is also small, the approximation will be worse. In the worst case when the minimum fabrication width is large and the expansion ratio is small, e.g., $w_{\min }=2 \mathrm{~mm}$ and $R=0.8$ in Fig.11, the circle is approximated by a triangle. This study shows that the developed simulation framework can validate the material design and generate the related fabrication process plan as well.

\section{Conclusion}

The growth of 4D printing demonstrates the importance of expanding the concept of fabrication process to include time as a design factor. This paper introduces GeometryDriven Finite Elements (GDFE) as a new simulation and process planning framework for the 4D printing process based on thermal-shrinking structures. GDFE simplifies the mathematical model of accounting for complex sources that may cause the deformation of a structure into a geometric optimization problem. The GDFE framework provides an intuitive and effective way to enable simulation and planning for 4D printing even when a complete mathematical model of new material is not available yet. The experimental studies of the crease-fold design are reanalyzed, and the deformation principles are induced for the thick-fold design. In this paper, the negative thermal expansion ratio is used as the deformation principle. The deformation is applied on each GDFE, which is then assembled into a larger system that describes the overall deformed shape. The framework is validated by repeating the crease-fold designs as well as generating freeform shapes. A number of applications enabled by the GDFE framework have also been shown.

\subsection{Future Work}

The 2D-to-3D shape-shifting behaviors are studied in this paper, a more challenging case is the evolution of 3D structures. We will study the deformation principles of and extend this framework to 3D-to-3D deformations in the near future. While the $1 \mathrm{D}$ case can be designed inversely by mathematical expression of geometric primitives, it is much more challenging for a 2D-to-3D and 3D-to-3D cases. The inverse problem is far more complex in determining a discrete smallscale material distribution that yields the desired self-folding behavior. In order to solve this problem, we are studying a new pattern learning and synthesis framework with the data-driven approaches. Another future work is to apply the GDFE framework to more complex deformation principle other than expansion or contraction.

\section{Acknowledgements}

We acknowledge the support of the Natural Sciences \& Engineering Research Council of Canada (NSERC) grant \# RGPIN-2017-06707, the USC Epstein Institute and the USC Northrop Grumman Institute of Optical Nanomaterials and Nanophotonics.

\section{References}

[1] Hull, C. W., 1984. Apparatus for production of three-dimensional objects by stereolithography. United States patent US 4,575,330.

[2] Park, J.-R., Slanac, D. A., Leong, T. G., Ye, H., Nelson, D. B., and Gracias, D. H., 2008. "Reconfigurable microfluidics with metallic containers". Journal of Microelectromechanical Systems, 17(2), April, pp. 265-271.

[3] Azam, A., Laflin, K. E., Jamal, M., Fernandes, R., and Gracias, D. H., 2011. "Self-folding micropatterned polymeric containers". Biomedical Microdevices, 13(1), pp. 51-58.

[4] Gao, W., Zhang, Y., Ramanujan, D., Ramani, K., Chen, Y., Williams, C. B., Wang, C. C., Shin, Y. C., Zhang, S., and Zavattieri, P. D., 2015. "The status, challenges, and future of additive manufacturing in engineering". Comput. Aided Des., 69(C), Dec., pp. 65-89.

[5] Lang, R. J., 2011. Origami Design Secrets: Mathematical Methods for an Ancient Art. CRC Press, Boca Raton, FL.

[6] Zhang, K., Qiu, C., and Dai, J. S., 2015. "Helical kirigami-enabled centimeter-scale worm robot with shape-memory-alloy linear actuators". Journal of Mechanisms and Robotics, 7(2), p. 021014.

[7] Ge, Q., Qi, H. J., and Dunn, M. L., 2013. "Active materials by four-dimension printing". Applied Physics Letters, 103(13), p. 131901.

[8] Tibbits, S., 2014. "4D printing: Multi-material shape change". Architectural Design, 84(1), pp. 116-121.

[9] Deng, D., and Chen, Y., 2015. "Origami-based selffolding structure design and fabrication using projection based stereolithography". J. Mech. Des., 137(2), p. 021701:12.

[10] Sydney Gladman, A., Matsumoto, E. A., Nuzzo, R. G., Mahadevan, L., and Lewis, J. A., 2016. "Biomimetic 4d printing”. Nature Materials, 15(4), pp. 413 - 418.

[11] Felton, S., Tolley, M., Demaine, E., Rus, D., and Wood, R., 2014. "A method for building self-folding machines". Science, 345(6197), pp. 644-646.

[12] Na, J.-H., Evans, A. A., Bae, J., Chiappelli, M. C., Santangelo, C. D., Lang, R. J., Hull, T. C., and Hayward, R. C., 2015. "Programming reversibly self-folding origami with micropatterned photo-crosslinkable polymer trilayers". Advanced Materials, 27(1), pp. 79-85.

[13] Breger, J. C., Yoon, C., Xiao, R., Kwag, H. R., Wang, M. O., Fisher, J. P., Nguyen, T. D., and Gracias, D. H., 2015. "Self-folding thermo-magnetically responsive soft microgrippers". ACS Applied Materials \& Interfaces, 7(5), pp. 3398-3405.

[14] Geryak, R., and Tsukruk, V. V., 2014. "Reconfigurable 
and actuating structures from soft materials". Soft Matter, 10, pp. 1246-1263.

[15] Malachowski, K., Breger, J., Kwag, H. R., Wang, M. O., Fisher, J. P., Selaru, F. M., and Gracias, D. H., 2014. "Stimuli-responsive theragrippers for chemomechanical controlled release". Angewandte Chemie International Edition, 53(31), pp. 8045-8049.

[16] Kwok, T.-H., Wang, C. C. L., Deng, D., Zhang, Y., and Chen, Y., 2015. "Four-dimensional printing for freeform surfaces: Design optimization of origami and kirigami structures". J. Mech. Des., 131(1), p. 111413:10.

[17] Hernandez, E. A. P., Hartl, D. J., Akleman, E., and Lagoudas, D. C., 2016. "Modeling and analysis of origami structures with smooth folds". ComputerAided Design, 78, pp. 93 - 106. \{SPM\} 2016.

[18] Momeni, F., Hassani.N, S. M., Liu, X., and Ni, J., 2017. "A review of $4 \mathrm{~d}$ printing". Materials \& Design, 122, pp. $42-79$.

[19] Tibbits, S., 2012. "Design to self-assembly". Architectural Design, 82(2), pp. 68-73.

[20] Khoo, Z. X., Teoh, J. E. M., Liu, Y., Chua, C. K., Yang, S., An, J., Leong, K. F., and Yeong, W. Y., 2015. "3d printing of smart materials: A review on recent progresses in 4d printing". Virtual and Physical Prototyping, 10(3), pp. 103-122.

[21] Choi, J., Kwon, O.-C., Jo, W., Lee, H. J., and Moon, M.-W., 2015. "4d printing technology: A review". 3D Printing and Additive Manufacturing, 2(4), pp. 159167.

[22] Wang, M.-F., Maleki, T., and Ziaie, B., 2008. "Enhanced 3-D folding of silicon microstructures via thermal shrinkage of a composite organic/inorganic bilayer". Journal of Microelectromechanical Systems, 17(4), Aug, pp. 882-889.

[23] Yasu, K., and Inami, M., 2012. "Popapy: Instant paper craft made up in a microwave oven". In Advances in Computer Entertainment, A. Nijholt, T. Romo, and D. Reidsma, eds., Vol. 7624 of Lecture Notes in Computer Science. Springer Berlin Heidelberg, pp. 406420.

[24] Smela, E., 2003. "Conjugated polymer actuators for biomedical applications". Advanced Materials, 15(6), pp. 481-494.

[25] Ionov, L., 2012. "Biomimetic 3D self-assembling biomicroconstructs by spontaneous deformation of thin polymer films". J. Mater. Chem., 22, pp. 19366-19375.

[26] Peraza-Hernandez, E., Hartl, D., Galvan, E., and Malak, R., 2013. "Design and optimization of a shape memory alloy-based self-folding sheet". Journal of Mechanical Design, 135, p. 111007.

[27] Ionov, L., 2011. "Soft microorigami: self-folding polymer films". Soft Matter, 7, pp. 6786-6791.

[28] Shim, T. S., Kim, S.-H., Heo, C.-J., Jeon, H. C., and Yang, S.-M., 2012. "Controlled origami folding of hydrogel bilayers with sustained reversibility for robust microcarriers". Angewandte Chemie International Edition, 51(6), pp. 1420-1423.
[29] Stoychev, G., Turcaud, S., Dunlop, J. W. C., and Ionov, L., 2013. "Hierarchical multi-step folding of polymer bilayers". Advanced Functional Materials, 23(18), pp. 2295-2300.

[30] Ahmed, S., Lauff, C., Crivaro, A., McGough, K., Sheridan, R., Frecker, M., von Lockette, P., Ounaies, Z., Simpson, T., Lien, J.-M., and Strzelec, R., 2013. "Multi-field responsive origami structures: Preliminary modeling and experiments". In Proceedings of the ASME IDETC/CIE, August 4-7, Portland, Oregon, USA, p. V06BT07A028.

[31] Liu, Y., Boyles, J. K., Genzer, J., and Dickey, M. D., 2012. "Self-folding of polymer sheets using local light absorption”. Soft Matter, 8, pp. 1764-1769.

[32] Raviv, D., Zhao, W., McKnelly, C., Papadopoulou, A., Kadambi, A., Shi, B., Hirsch, S., Dikovsky, D., Zyracki, M., Olguin, C., Raskar, R., and Tibbits, S., 14. "Active printed materials for complex self-evolving deformations". Sci. Rep., 4.

[33] Schenk, M., and Guest, S. D. ., 2011. "Origami folding: A structural engineering approach". In Origami 5: Fifth International Meeting of Origami Science, Mathematics, and Education, p. 291303.

[34] Tachi, T., 2013. "Interactive form-finding of elastic origami". In the International Association for Shell and Spatial Structures (IASS) Symposium.

[35] Zhu, L., Igarashi, T., and Mitani, J., 2013. "Soft folding". Computer Graphics Forum, 32(7), pp. 167-176.

[36] Belcastro, S.-M., and Hull, T. C., 2002. "Modelling the folding of paper into three dimensions using affine transformations". Linear Algebra and its Applications, 348(13), pp. 273 - 282.

[37] Tachi, T., 2010. Advances in Architectural Geometry. Springer Vienna, Vienna, ch. Freeform RigidFoldable Structure using Bidirectionally Flat-Foldable Planar Quadrilateral Mesh, pp. 87-102.

[38] Hwang, H.-D., and Yoon, S.-H., 2015. "Constructing developable surfaces by wrapping cones and cylinders". Computer-Aided Design, 58, pp. 230 - 235. Solid and Physical Modeling 2014.

[39] Pan, Y., Zhou, C., and Chen, Y., 2012. "A fast mask projection stereolithography process for fabricating digital models in minutes". Journal of Manufacturing Science and Engineering, 134(5), pp. 051011 9.

[40] Zhou, C., Chen, Y., Yang, Z., and Khoshnevis, B., 2013. "Digital material fabrication using maskimageprojectionbased stereolithography". Rapid Prototyping Journal, 19(3), pp. 153-165.

[41] Bodansky, E., and Gribov, A., 2006. Approximation of a Polyline with a Sequence of Geometric Primitives. Springer Berlin Heidelberg, Berlin, Heidelberg, pp. 468-478. 